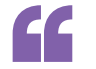

...it is very challenging to detect subsurface defects or measure the critical dimensions of substructures...

$\Rightarrow$ ELECTRONIC DEVICES

\title{
Finding flaws in a flash
}

Flash memory devices based on negative-AND (NAND) logic gates are important data storage components of USB drives, solid-state drives, smart phones, computers, servers and cars. The devices are non-volatile, high density and low cost; however, to improve the yield of the devices, more details about the defects within them are needed. The yield is considered to be the ratio of working devices with good performance that are defect-free, compared to the total number of devices fabricated on a single Si wafer.

Now, writing in Nature Electronics, Seok Ho Song, Seong-Min Ma and colleagues use optical detection methods to identify subsurface defects in NAND flash memory devices. To achieve this, they consider the hyperbolic metamaterial structure of the $3 \mathrm{D}$ devices and, more specifically, the propagation of optical states that exist as hyperbolic Bloch modes and the optical interaction of these materials with plasmonic resonances. These Bloch modes have characteristic features in the near-IR and this phenomenon allows resonantly enhanced optical fields to penetrate the full depth of the device (that is, on the micrometre scale).

NAND flash devices have vertical stacks composed of metal-dielectricsemiconductor composites with periodic slits separating the stacks. In the devices fabricated by the researchers, these stacks comprise hybrid $\mathrm{W}-\mathrm{SiO}_{2}$ layers, with internal holes containing concentric cylinders of $\mathrm{SiO}_{2}-\mathrm{Si}_{2} \mathrm{~N}_{3}$ $\mathrm{SiO}_{2}$-poly-Si-SiO 2 , in which $\mathrm{Si}_{2} \mathrm{~N}_{3}$ are the data storage rings and poly-Si acts as access channels. Each W layer is called a wordline and the $\mathrm{SiO}_{2}$ layer between neighbouring wordlines has the effect of reducing the cross-talk effect in the devices.

The production of these devices requires hundreds of processing steps and, as a consequence, defects are expected. "However, it is very challenging to detect subsurface defects or measure the critical dimensions of substructures due to the lack of optical penetration into the depths

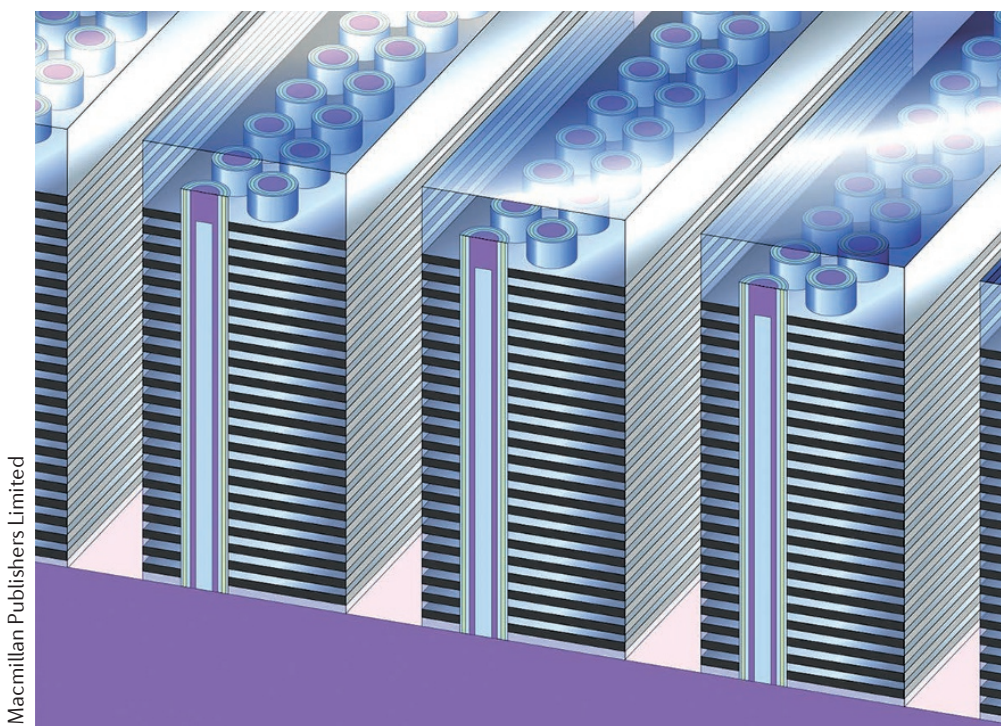

of the structure," says Ma. Most commonly, these defects are voids buried in the stacks, which may cause an open-circuit failure, and $\mathrm{W}$ bridges between wordlines, which may cause short-circuit failure. Also, W residues at the bottom of the slits between the wordlines and failure of substitution to give a $\mathrm{W}-\mathrm{SiO}_{2}$ layer are other sources of defects in the devices.

Using a bright-field optical microscopy method with a broadband light (near-IR to IR) source, the researchers show how a diffraction-assisted resonance (or a diffraction-assisted volume-plasmonic resonance near an epsilon-near-zero condition) enables the detection of defects at a depth almost ten times deeper than the conventional optical limit.

This information about subsurface defects is becoming more important as the sizes of state-ofthe-art devices increases. At present, fabrication methods allow $72 \mathrm{~W}-\mathrm{SiO}_{2}$ stacks on a patterned Si wafer, and this number is likely to increase to enable a higher density of data to be obtained. In addition, films with different compositions may have different compressive or tensile stresses and, as a result, the combinations of these films may cause severe warpage of the wafer or irregular patterns on the wafer. "Thus, it will be challenging to establish stable, mass-production process steps with improved etch and deposition technologies, as well as better control the accumulated stresses of the deposited films," concludes Song.

Alison Stoddart

ORIGINAL ARTICLE Yoon, J. W. et a.

Nanophotonic identification of defects buried in three-dimensional NAND flash memory devices. Nat. Electron. https://doi.org/10.1038/s41928-017. 0007-7 (2018) 